\title{
A systematic review and meta-analysis of the influence of STAS on the long-term prognosis of stage I lung adenocarcinoma
}

\author{
Yanhui Yang, Xiaoyang Xie, Yi Wang, Xiaoliang Li, Lei Luo, Yi Yao, Ji Li \\ Department of Thoracic Surgery, The First People's Hospital of Neijiang, Neijiang, China \\ Contributions: (I) Conception and design: Y Yang; (II) Administrative support: J Li; (III) Provision of study materials or patients: Y Wang; (IV) \\ Collection and assembly of data: Y Yao; (V) Data analysis and interpretation: Y Yao; (VI) Manuscript writing: All authors; (VII) Final approval of \\ manuscript: All authors. \\ Correspondence to: Ji Li. Department of Thoracic Surgery, The First People’s Hospital of Neijiang, Neijiang, China. Email: Jimlee0832@163.com.
}

Background: The appearance of airway dissemination often indicates poor prognosis of lung cancer. However, the relationship between airway dissemination and early lung adenocarcinoma is not clear. Therefore, this study uses meta-analysis to study the long-term prognosis of patients with early lung adenocarcinoma.

Methods: A computer search of the PubMed, EMbase, Web of Science, and other databases was conducted, and the search time limit was from the establishment of the database to December 30, 2020. Data strictly in accordance with the inclusion and exclusion criteria was extracted and the quality of the included literature was evaluated. Two reviewers then independently screened the literature and evaluated the risk of bias.

Results: A total of 11 studies were included, comprised of 5,097 patients with early lung adenocarcinoma. The results of the meta-analysis showed that among patients with stage I lung adenocarcinoma, the 5-year recurrence-free survival (RFS) and overall survival (OS) of STAS-positive patients was lower than those of the STAS-negative group, and the difference was statistically significant. In the control study, the 5-year RFS was HR $=1.95,95 \%$ CI $(1.58-2.31) \mathrm{P}<0.01$, and the 5 -year OS was OR $=2.04$, 95\% CI (1.60-2.48) $\mathrm{P}<0.01$, and in STAS-positive patients, sublobectomy had a worse long-term prognosis than lobectomy. While the 5-year RFS HR of the lobectomy group was 1.82, 95\% CI (1.43-2.22), and the 5-year RFS HR of the sublobectomy group was $6.92,95 \%$ CI $(1.64-12.18) \mathrm{P}<0.01$, the prognosis of the STAS-positive highexpression group and the low-expression group was worse. The 5-year RFS HR of the low-expression STAS group was 2.93, 95\% CI (0.21-6.07), and in the high expression group this was $8.20,95 \%$ CI $(0.55-15.85)$ $\mathrm{P}<0.05$.

Discussion: STAS is an independent risk factor for the poor prognosis of stage I lung adenocarcinoma and a high expression of STAS results in a higher 5-year recurrence rate. When STAS is positive, the sublobectomy method should be carefully selected. However, our research has certain limitations, such as literature selection and publication bias. At the same time, the depth of literature analysis needs to be further improved.

Keywords: STAS; long-term prognosis; stage I lung adenocarcinoma; meta-analysis

Submitted Mar 22, 2021. Accepted for publication May 18, 2021.

doi: $10.21037 /$ tcr-21-750

View this article at: http://dx.doi.org/10.21037/tcr-21-750 


\section{Introduction}

The phenomenon of STAS (airway spread through air space) was proposed by the World Health Organization in 2015 and is considered a new invasion mode (1) which specifically refers to micropapillary clusters, solid cancer suppression, or single cells outside the tumor entering the surrounding parenchyma through the airway in the alveoli. Although studies have shown that STAS is correlated with a poor prognosis of lung cancer, the specific prognostic mode and mechanism are not clear (2). Current literature confirms that STAS is more common in patients with lung adenocarcinoma, which is replacing squamous cell carcinoma as the most common in this country. Surgery is an effective treatment for early lung cancer and most patients with stage I lung adenocarcinoma obtain a satisfactory prognosis after effective surgical intervention. However, the 5 -year survival rate in some patients remains less than $50 \%$ (3). With developments in precision medicine and thoracoscopic surgery, sublobectomy represented by anatomical segmentectomy has gradually replaced anatomical lobectomy, especially for the treatment of early lung cancer, in patients with poor cardiopulmonary function, when the lesion diameter is less than $2 \mathrm{~cm}$, and in elderly patients (4). However, whether in pure ground glass nodules or solid nodules, airway dissemination may occur, which raises the question of whether the poor prognosis of early lung adenocarcinoma is related to the STAS. This study used three online database systems to analyze related studies published before 2020, and a systematic review and metaanalysis were conducted to provide clinicians with certain references for early lung cancer surgical selection and clinical diagnosis and treatment. We present the following article in accordance with the PRISMA reporting checklist (available at http://dx.doi.org/10.21037/tcr-21-750).

\section{Methods}

\section{Literature inclusion and exclusion criteria}

\section{Study type}

We compared the 5-year recurrence-free survival (RFS) and overall survival (OS) of STAS positive and STAS negative patients with stage I lung adenocarcinoma. Randomized controlled trials (RCTs), cohort studies, and randomized controlled studies published in English were evaluated.

\section{Research subjects}

Patients with stage I lung adenocarcinoma who had undergone thoracotomy and thoracoscopic lobectomy or sublobectomy (pulmonary wedge, lung segment), regardless of race, nationality, gender, and age.

\section{Intervention measures}

STAS positive patients were placed in the experimental group and STAS negative patients in the control group. The specific surgical methods utilized were then described.

\section{Outcome indicators}

The 5-year RFS and OS between the overall and different subgroups were used.

\section{Exclusion criteria}

(I) Non-English literature; (II) repeated literature; (III) patients with other tumors; (IV) patients without relevant outcome indicators (non-phase I studies or lack of phase I studies).

\section{Literature search}

Two independent researchers searched the PubMed, EMbase, Web of Science, and Cochrane Library databases and the search time was from the establishment of the library to December 2020. The search terms used included the following: STAS (spread through air space, spread through air spaces), lung cancer, lung neoplasm, lung carcinoma, and non-small cell lung cancer (NSCLC). Reference lists were also checked for relevant articles.

\section{Literature screening and quality evaluation}

Two independent researchers screened the literature, extracted data, and cross-checked and disagreement was resolved by consultation and discussion with a third party. In cases where information was missing, attempts were made to contact the original author. When selecting documents, the title and abstract were first read, and after excluding obviously irrelevant documents, the full text was read to further determine eligibility. When the HR value was not provided in the literature, but the survival curve was available, the Engague Digitizer was used to determine the coordinates to obtain relevant data. The data extraction content mainly included the following: (I) the basic information of the included research, the first author, and the publication time; (II) the baseline characteristics of the research object including the number of samples in 


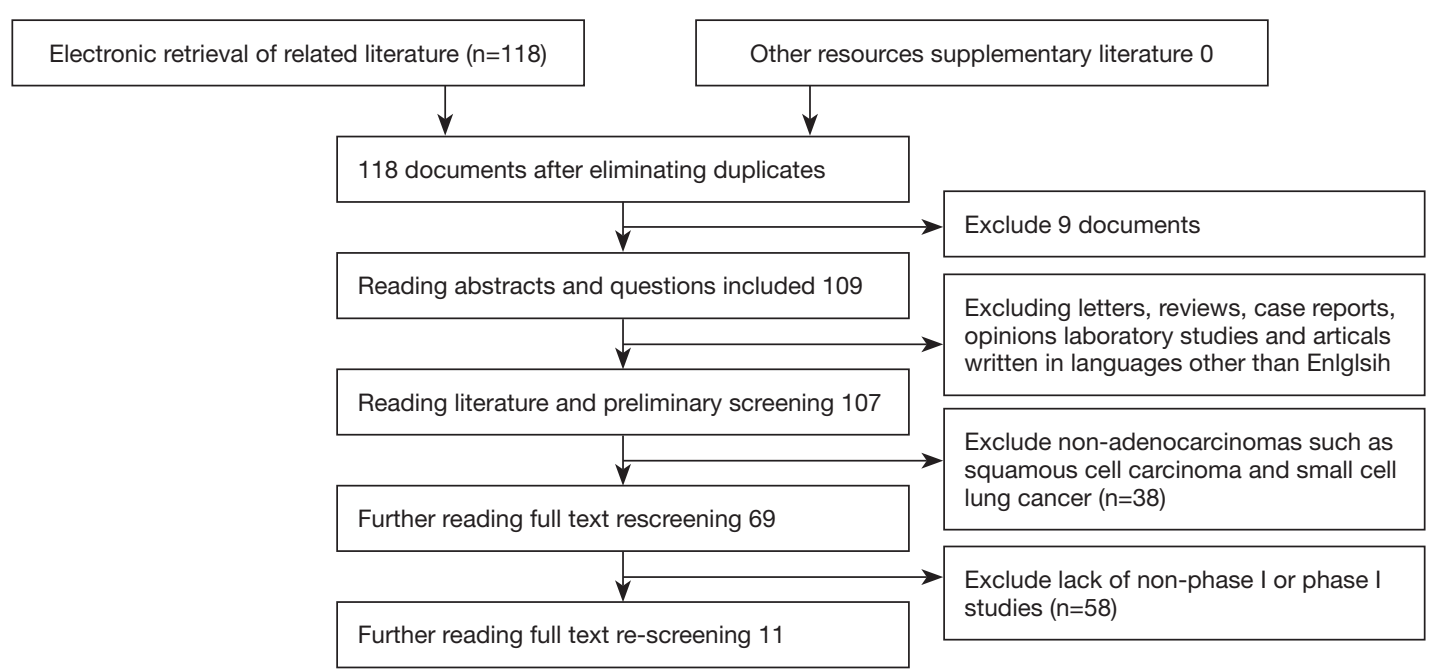

Figure 1 Document screening flowchart.

each group and the age of patients; (III) the specific details of intervention measures; (IV) the key elements of bias risk evaluation; and $(\mathrm{V})$ the outcome indicators of concern.

Evaluation of the risk of bias of the included studies according to the Newcastle-Ottawa Scale (NOS) to evaluate the risk of bias of cohort studies and case-control studies, was determined according to the Cochrane System Reviewer's Manual for randomized controlled trials (RCT) bias risk evaluation tool Evaluate the risk of bias.

\section{Statistical analysis}

Stata 12.0 software was used for the meta-analysis and using HR as the effect indicator, each effect size was given its point estimate and $95 \%$ CI. Heterogeneity among the results of the included studies was analyzed by the $\chi^{2}$ test (the test level is $\alpha=0.1$ ), and $\mathrm{I}^{2}$ was used to judge the degree of heterogeneity between the studies. If $\mathrm{I}^{2}<50 \%$, the fixed-effects model was used for meta-analysis and if $\mathrm{I}^{2}>50 \%$, the subgroup analysis was used to find the source of heterogeneity. If there was no exact source, the randomeffects model was used. The test level was set to $\alpha=0.05$, and in cases of obvious clinical heterogeneity, subgroup analysis or MetaAnalyst software was used to conduct sensitivity analysis of the impact of a single study on the overall study.

\section{Results}

\section{Literature screening process and results}

A total of 118 related publications comprising 5,097 patients with stage 1 lung adenocarcinoma were obtained, and 11 publications were included in the final analysis. The specific literature screening process is shown in Figure 1.

\section{Meta-analysis results}

\section{5-year RFS and 5-year OS}

The 11 documents reported on 14 studies detailing the 5 -year RFS of patients with stage I lung adenocarcinoma and there was no significant heterogeneity among the included studies in RFS $\left(\mathrm{I}^{2}=16.2 \%, \mathrm{P}=0.276\right)$ and $\mathrm{OS}$ $\left(\mathrm{I}^{2}=0.0 \%, \mathrm{P}=0.967\right)$. Overall, STAS-positive stage I lung adenocarcinoma patients had no recurrence in 5 years, and the survival and 5-year OS were lower than those of STASnegative stage I lung adenocarcinoma patients, see Figure 2 for details.

A total of six documents reported 5-year RFS and OS of patients with stage I lung adenocarcinoma who received standard anatomical lobectomy. The results showed that there was no significant heterogeneity in RFS $\left(\mathrm{I}^{2}=22.2 \%\right.$, $\mathrm{P}=0.267)$, combined effect size HR $=1.82,95 \%$ CI $(1.43-$ 2.22), and OS, and there was no significant heterogeneity between the included studies $\left(\mathrm{I}^{2}=0.0 \%, \mathrm{P}=0.828\right) .5$-year RFS and 5-year OS of STAS-positive stage I lung adenocarcinoma were lower than those of STAS-negative stage I lung adenocarcinoma, see Figure 3 for details.

Two articles reported the 5-year RFS and OS of patients with stage I lung adenocarcinoma undergoing sublobectomy. And there was no significant heterogeneity between the studies $\left(\mathrm{I}^{2}=0.0 \%, \mathrm{P}=0.374\right)$. The combined 


\begin{tabular}{|c|c|c|c|}
\hline $\begin{array}{l}\text { Study } \\
\text { ID }\end{array}$ & & $\mathrm{ES}(95 \% \mathrm{Cl})$ & $\begin{array}{l}\% \\
\text { weight }\end{array}$ \\
\hline RFS & & & \\
\hline Eunjue Yi (2020) & & $3.49(0.77,15.80)$ & 0.23 \\
\hline Gouji Toyokawa (2018) & & $9.74(1.78,18.12)$ & 0.20 \\
\hline Yijiu Ren (2019) & $\approx$ & $3.89(1.11,5.63)$ & 2.59 \\
\hline Yijiu Ren (2019) & $\rightarrow$ & $4.89(1.84,15.65)$ & 0.28 \\
\hline Yifan Zhong (2020) & - & $1.87(1.28,2.74)$ & 24.67 \\
\hline Kyuichi Kadota (2019) & & $6.87(3.08,15.31)$ & 0.35 \\
\hline Satoshi Shiono (2016) & - & $2.24(1.36,3.71)$ & 9.57 \\
\hline Lin Yang (2018) & 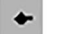 & $1.75(0.96,3.19)$ & 10.61 \\
\hline Donglai Chen (2020) & 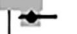 & $3.31(1.62,6.80)$ & 1.97 \\
\hline Yeon Bi Han (2020) & $1-$ & $3.44(1.35,8.79)$ & 0.95 \\
\hline Yeon Bi Han (2020) & & $8.43(3.44,20.62)$ & 0.18 \\
\hline Chenyang Dai (2017) & - & $1.66(1.02,2.07)$ & 47.96 \\
\hline Hironori Uruga (2017) & $\infty$ & $1.65(0.23,11.97)$ & 0.38 \\
\hline Hironori Uruga (2017) & & $7.35(1.53,35.17)$ & 0.05 \\
\hline Subtotal $(I-$ squared $=16.2 \%, P=0.276)$ & 1 & $1.95(1.58,2.31)$ & 100.00 \\
\hline os & & & \\
\hline Eunjue Yi (2020) & $\leftarrow$ & $1.33(0.35,5.11)$ & 3.40 \\
\hline Gouji Toyokawa (2018) & 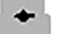 & $1.98(1.08,3.63)$ & 11.86 \\
\hline Yijiu Ren (2019) & - & $1.85(1.12,3.04)$ & 20.92 \\
\hline Yijiu Ren (2019) & & $7.11(1.96,25.87)$ & 0.13 \\
\hline Yifan Zhong (2020) & - & $2.06(1.30,3.27)$ & 19.82 \\
\hline Kyuichi Kadota (2019) & $\infty$ & $2.85(1.59,5.12)$ & 6.19 \\
\hline Satoshi Shiono (2016) & 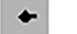 & $2.40(1.45,3.93)$ & 12.54 \\
\hline Lin Yang (2018) & $\rightarrow$ & $3.92(1.60,9.61)$ & 1.20 \\
\hline Donglai Chen (2020) & $1 \rightarrow$ & $3.46(1.26,9.51)$ & 1.13 \\
\hline Yeon Bi Han (2020) & 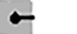 & $0.99(0.27,3.60)$ & 6.98 \\
\hline Yeon Bi Han (2020) & $\leftarrow$ & $1.60(0.43,5.95)$ & 2.53 \\
\hline Chenyang Dai (2017) & 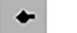 & $2.10(1.21,3.65)$ & 12.96 \\
\hline Hironori Uruga (2017) & & $2.49(0.15,41.70)$ & 0.04 \\
\hline Hironori Uruga (2017) & مـ & $2.91(0.50,17.05)$ & 0.28 \\
\hline Subtotal $(\mathrm{I}$-squared $=0.0 \%, P=0.967)$ & 4 & $2.04(1.60,2.48)$ & 100.00 \\
\hline 1 & & & \\
\hline-41.7 & 0 & .7 & \\
\hline
\end{tabular}

Figure 25 -year recurrence-free survival and 5-year overall survival.

effect size of 5 -year RFS was HR $=6.92,95 \%$ CI $(1.64-$ 12.18), and the 5 -year RFS of STAS-positive stage I lung adenocarcinoma was lower than that of STAS-negative stage I lung adenocarcinoma.

In stage I lung adenocarcinoma patients with low STAS expression (stas1), there were two articles reporting 5-year RFS and OS and no significant heterogeneity was seen among the studies. The corresponding RFS heterogeneity were $\left(\mathrm{I}^{2}=0, \mathrm{P}=0.613\right)$, and combined 5-year RFS effect size were HR 2.931, 95\% CI (0.212-6.074).

In stage I lung adenocarcinoma with high STAS expressed there was no significant heterogeneity between the studies. The corresponding studies RFS heterogeneity were $\left(\mathrm{I}^{2}=0, \mathrm{P}=0.911\right)$, and the combined effect (RFS) size was HR 8.20, 95\% CI (0.55-15.85). See Figure 4 for details.

\section{Features of included articles}

The basic features of included articles are shown in Table 1 .

\section{Analysis of publication bias}

Using stata software to construct a Begg chart and analysis, the test results showed that no publication bias was found in 5 -year RFS $(\mathrm{P}=0.003)$ and 5 -year OS $(\mathrm{P}=0.951)$, as shown in Figure 5 .

\section{Discussion}

Lung adenocarcinoma is a special pathological type of NSCLC, the incidence and mortality rate of which has been increasing in recent years. There are many factors that affect the long-term survival of lung cancer patients, such as TNM staging, tumor differentiation degree, postoperative complications, and postoperative adjuvant treatment. An increasing awareness of the benefits of screening has resulted in the detection of many early lung cancers and effective surgical intervention. However, even after the effective surgical resection of early lung cancer 


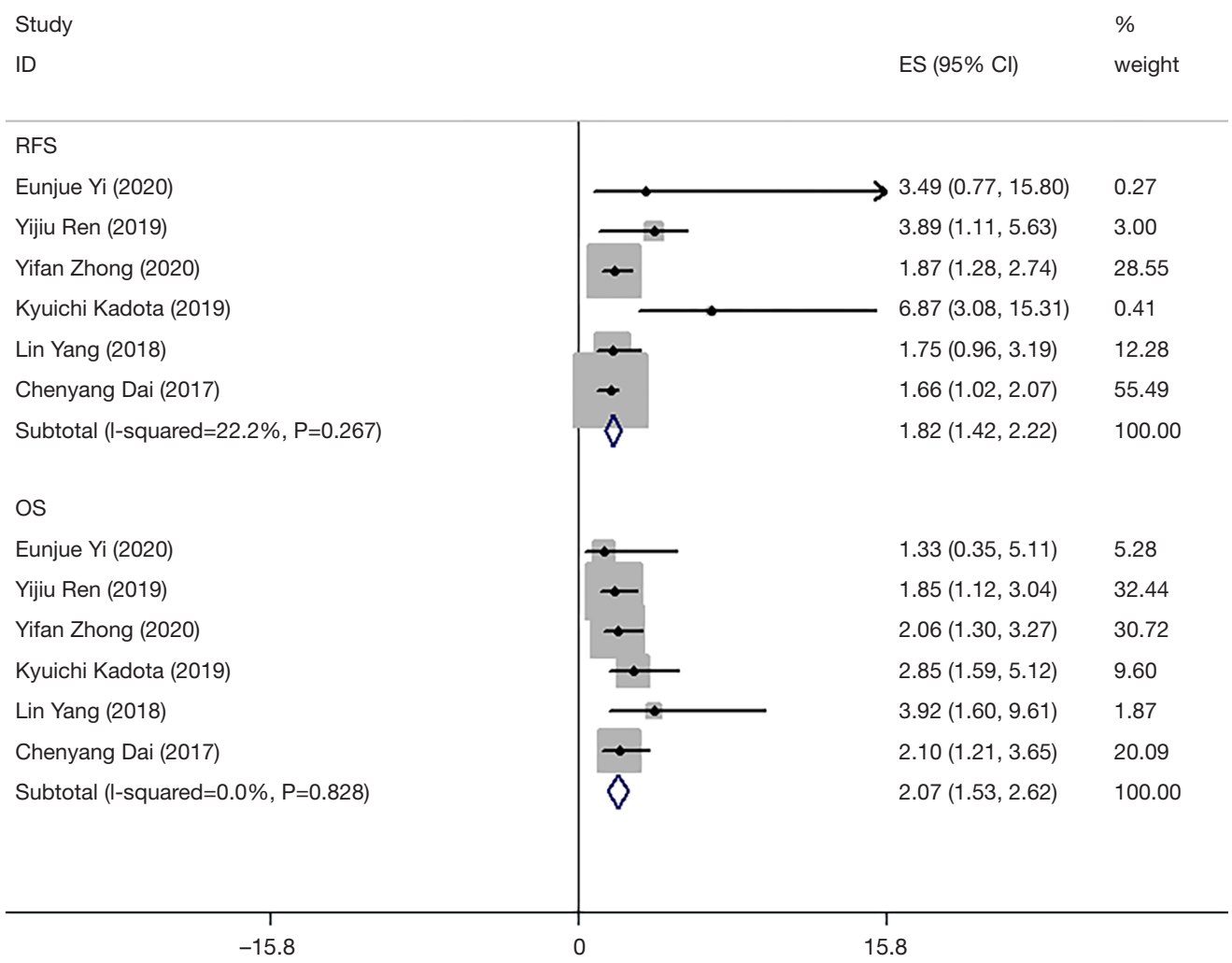

Figure 3 5-year RFS and 5-year OS in the lobectomy group. RFS, recurrence-free survival; OS, overall survival.

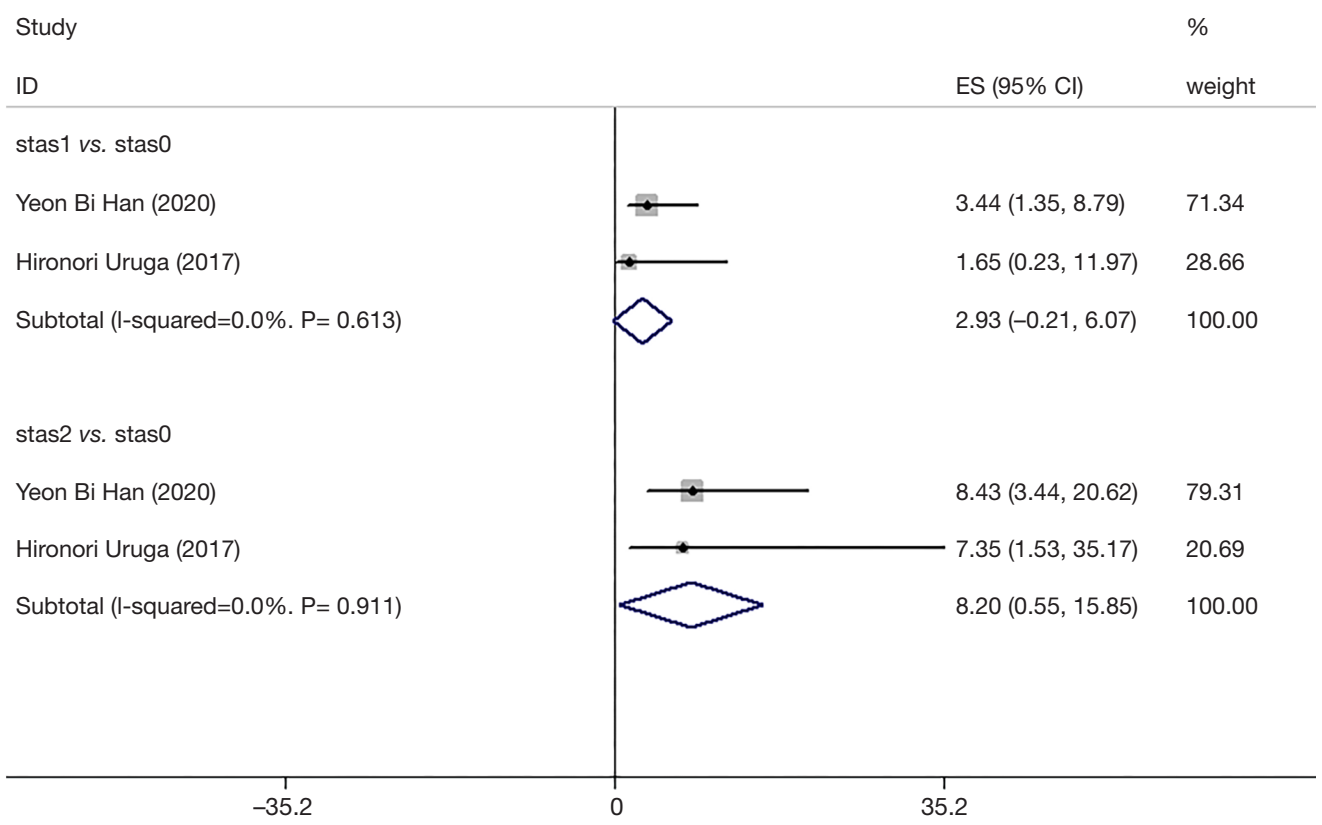

Figure 4 Forest plot of high STAS expression (stas2) and low STAS expression (stas1). 
Table 1 Basic characteristics of included articles

\begin{tabular}{|c|c|c|c|c|c|c|c|c|}
\hline Study & Year & Operative type & $\begin{array}{l}\text { Study } \\
\text { region }\end{array}$ & $\begin{array}{l}\text { Median } \\
\text { age }\end{array}$ & Type & Outcome & $\begin{array}{l}\text { NOS } \\
\text { score }\end{array}$ & $\mathrm{HR}$ \\
\hline Satoshi Shiono & 2016 & Lob and sub & Japan & 70 & $A D C$ & RFS, OS & 7 & $\begin{array}{l}\text { 5-year RFS: HR =2.24, 95\% Cl }(1.36-3.71) \\
\text { 5-year OS: } \mathrm{HR}=2.4,95 \% \mathrm{Cl}(1.45-3.93)\end{array}$ \\
\hline Chenyang Dai & 2017 & Lobectomy & China & 60 & $A D C$ & RFS, OS & 7 & $\begin{array}{l}\text { 5-year RFS: HR =1.66, 95\% Cl }(1.02-2.07) \text {; } \\
\text { 5-year OS: HR =2.1, 95\% Cl (1.21-3.65) }\end{array}$ \\
\hline Hironori Uruga [2] & 2017 & Lob and sub & Japan & 66 & $A D C$ & RFS, OS & 7 & $\begin{array}{l}\text { 5-year RFS: HR =7.35, 95\% Cl }(1.54-35.17) \text {; } \\
\text { 5-year OS: HR }=2.91,95 \% \mathrm{Cl}(0.5-17.05)\end{array}$ \\
\hline Gouji Toyokawa & 2018 & Sub lobectomy & Japan & 71 & $A D C$ & RFS, OS & 7 & $\begin{array}{l}\text { 5-year RFS: HR =9.74, 95\% Cl }(1.78-18.12) \text {; } \\
\text { 5-year OS: } \mathrm{HR}=1.98,95 \% \mathrm{Cl}(1.08-3.63)\end{array}$ \\
\hline Kyuichi Kadota & 2019 & Lobectomy & Japan & 68 & ADC & RFS, OS & 7 & $\begin{array}{l}\text { 5-year RFS: HR =6.87, 95\% Cl (3.08-15.31); } \\
\text { 5-year OS: HR =2.85, 95\% Cl (1.59-5.12) }\end{array}$ \\
\hline Lin Yang & 2019 & Lobectomy & China & 58.73 & ADC & RFS, OS & 6 & $\begin{array}{l}\text { 5-year RFS: HR =1.75, 95\% Cl }(0.96-3.19) \\
\text { 5-year OS: } \mathrm{HR}=3.92,95 \% \mathrm{Cl}(1.60-9.61)\end{array}$ \\
\hline Yifan Zhong & 2020 & Lobectomy & China & 59.6 & ADC & RFS, OS & 6 & $\begin{array}{l}\text { 5-year RFS: HR =1.87, 95\% Cl }(1.28-2.74) \\
\text { 5-year OS: } \mathrm{HR}=2.06,95 \% \mathrm{Cl}(1.3-3.27)\end{array}$ \\
\hline Donglai Chen & 2020 & Lob and sub & China & 60 & $A D C$ & RFS, OS & 7 & $\begin{array}{l}\text { 5-year RFS: HR =3.31, 95\% Cl (1.62-6.8); } \\
\text { 5-year OS: HR =3.46, 95\% Cl (1.26-9.51) }\end{array}$ \\
\hline
\end{tabular}

ADC, adenocarcinoma; RFS, recurrence-free survival; OS, overall survival.

(stage I), the long-term prognosis is still unsatisfactory, with a 5 -year survival rate of $45-65 \%$ (5). In this study, a systematic evaluation of the long-term prognosis of earlystage lung adenocarcinoma and its association with the STAS phenomenon was conducted. The target population was fixed as early-stage and lung adenocarcinoma, mainly to minimize confounding factors that affect the long-term survival of lung cancer.

We conducted a meta-analysis of whether STAS occurred in early lung adenocarcinoma. A total of 5,097 patients were included and the results confirm that STAS is an independent risk factor for stage I lung adenocarcinoma recurrence and 5-year poor prognosis, HR $=1.95,95 \%$ CI (1.58-2.31), and these results are supported by those of several other studies. Yanagawa et al. (6) found that the incidence of STAS in the surgical resection of squamous cell carcinoma was $19.1 \%$ and was closely related to the 5 -year recurrence of stage I lung squamous cell carcinoma, but there was no statistical difference between stages II and III. Yang et al. (7) studied 242 cases of stage I lung adenocarcinoma and found that STAS was an independent risk factor for patients with a diameter of less than $2 \mathrm{~cm}$ who had undergone radical lobectomy. Shiono et al. (8) showed that STAS was closely related to lymphatic 


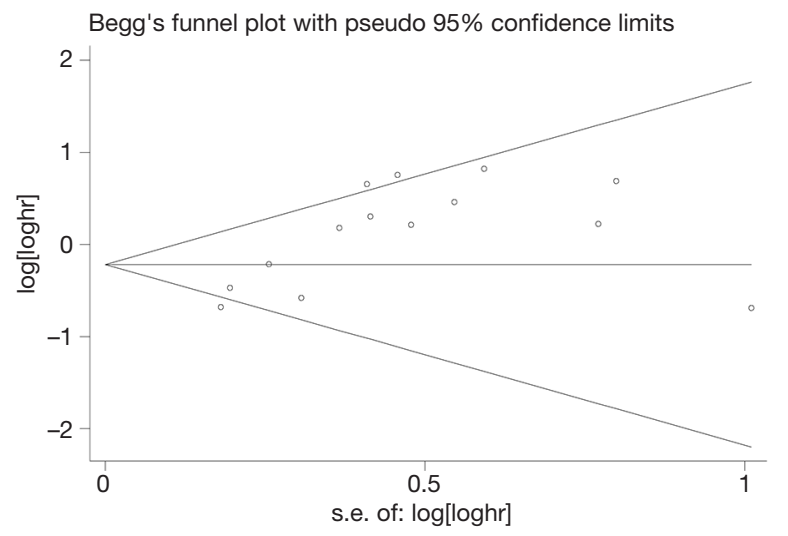

Figure 5 Publication bias analysis.

vessel invasion and pleural invasion in 318 cases of stage I lung adenocarcinoma, and the 5 -year survival rates of STAS cases were $62.7 \%$ and $91.1 \%(\mathrm{P}<0.01)$. Yi et al. (9) studied 109 cases of stage I lung adenocarcinoma and found that the presence of STAS may be an important risk factor for recurrence in patients with early aggressive lung adenocarcinoma treated by lobectomy. Through multivariate analysis Ren et al. (10) found that while STAS positive was not a predictor of adverse recurrence in a lobectomy group, it had a significant predictive effect on a sublobectomy group $(\mathrm{P}<0.001)$. Uruga et al. (11) studied 208 cases of lung adenocarcinoma and showed that STAS was closely related to shorter RFS and when comparing pleural invasion, nerve, and lymphatic invasion, multivariate analysis showed that STAS positive remained an important factor for poor prognosis. Finally, Dai et al. (12) showed that the expression of STAS correlated with tumor diameter.

The proportion of lung adenocarcinoma in overall lung cancer cases is increasing, and the OS from the disease remains low. Although molecular targeted therapy and immunosuppressive intervention have improved the longterm survival of patients with lung adenocarcinoma, lobectomy combined with lymph node dissection in the early stage of the disease remains the standard and most effective treatment option for patients. However, with the rapid development of thoracoscopy technology, some scholars have confirmed that for patients with lung adenocarcinoma with a diameter of $\leq 2 \mathrm{~cm}$ and a solid content of less than 25\%, sublobectomy (anatomical lung segment or lung wedge) resection) can not only achieve its radical purpose, but can also effectively preserve lung function and improve the patient's quality of life (13-16).
We speculate that STAS may complicate some patients undergoing this procedure, especially stage I patients, and are of the view this may account for the poor prognosis and recurrence of stage I lung adenocarcinoma. Our study included a total of 190 patients with subpulmonary resection and confirmed that sublobectomy is associated with a higher recurrence and worse 5 -year survival time when STAS is present RFS HR $=6.92,95 \%$ CI (1.64-12.18). This raises the question of whether stage 1 adenocarcinoma patients should continue to receive segmentectomy. At the same time, in anatomical segmentectomy, the surgeon often decides whether to replace lobectomy based on the condition of the hilar group of lymph nodes (11-14). Due to the cumbersome nature of lymph node examination and false negatives, some scholars suggest that the phenomenon of frozen STAS serves as the theoretical basis for anatomical lobectomy and postoperative adjuvant chemotherapy (17), although this may need to be confirmed by large-scale clinical studies.

Several researchers have investigated STAS since it was first defined and proposed by the World Health Organization, and several preoperative prediction systems have been proposed (18-21). Warth et al. (22) divided STAS into two groups based on the distance from the tumor, and Han et al. (23) divided the distance of STAS from the tumor into two levels. In these schemes, Grade I is within 2,500 $\mu \mathrm{m}$ from the edge of the tumor, and STAS from the edge of the tumor is equal to or greater than $2,500 \mu \mathrm{m}$. Grade II, STAS II had a worse long-term prognosis, regardless of the extent of resection. Toyokawa et al. (24) proposed an alternative classification method in a study of 110 cases of lung adenocarcinoma, according to the number of STAS spreading cells, namely no STAS, low STAS (1-4 cells or clusters), and high STAS ( $>4$ cells). In the specific grouping of early lung adenocarcinoma STAS, our study included two articles and 160 cases of stage I lung adenocarcinoma, which confirmed that the high STAS group had a worse 5-year RFS and OS. This finding inspires us to classify STAS with pathological characteristics as a topic for future research.

In summary, STAS is an independent risk factor for the poor prognosis of stage I lung adenocarcinoma, and a high expression of STAS has a higher 5-year recurrence rate. When STAS is positive, sublobectomy (segmentectomy or wedge resection) needs to be chosen carefully. Our metaanalysis has certain limitations. Firstly, the search was confined to publications in English, which may create a publication bias. For Begg's in the RFS group, there is a publication bias, $\mathrm{P}=0.003<0.05$. The possible reason for 
this may be that most of the research is Japanese and some is written by the same author. On this basis, publication bias is inevitable. Secondly, we only included stage I lung adenocarcinoma in our study, which may have created selection errors.

\section{Acknowledgments}

Funding: None.

\section{Footnote}

Reporting Checklist: The authors have completed the PRISMA reporting checklist. Available at http://dx.doi. org/10.21037/tcr-21-750

Conflicts of Interest: All authors have completed the ICMJE uniform disclosure form (available at http://dx.doi. org/10.21037/tcr-21-750). The authors have no conflicts of interest to declare.

Ethical Statement: The authors are accountable for all aspects of the work in ensuring that questions related to the accuracy or integrity of any part of the work are appropriately investigated and resolved.

Open Access Statement: This is an Open Access article distributed in accordance with the Creative Commons Attribution-NonCommercial-NoDerivs 4.0 International License (CC BY-NC-ND 4.0), which permits the noncommercial replication and distribution of the article with the strict proviso that no changes or edits are made and the original work is properly cited (including links to both the formal publication through the relevant DOI and the license). See: https://creativecommons.org/licenses/by-nc-nd/4.0/.

\section{References}

1. Travis WD, Brambilla E, Nicholson AG, et al. The 2015 World Health Organization Classification of Lung Tumors: Impact of Genetic, Clinical and Radiologic Advances Since the 2004 Classification. J Thorac Oncol 2015;10:1243-60.

2. Lee JS, Kim EK, Kim M, et al. Genetic and clinicopathologic characteristics of lung adenocarcinoma with tumor spread through air spaces. Lung Cancer 2018;123:121-6.

3. Ou SH, Zell JA, Ziogas A, et al. Prognostic factors for survival of stage I non-small lung cancer patients: a population-based analysis of 19702 stage I patients in the California Cancer Registry from 1989 to 2003. Cancer 2007;110:1532-41.

4. Shapiro M, Weiser TS, Wisnivesky JP, et al. Thoracoscopic segmentectomy compares favorably with thoracoscopic lobectomy for patients with small stage I lung cancer. J Thorac Cardiovasc Surg 2009;137:1388-93.

5. Raz DJ, Zell JA, Ou SH, et al. Natural history of stage I non small lung cancer: implications for early detection. Chest 2007;132:193-9.

6. Yanagawa N, Shiono S, Endo M, et al. Tumor spread through air spaces is a useful predictor of recurrence and prognosis in stage I lung squamous cell carcinoma, but not in stage II and III. Lung Cancer 2018;120:14-21.

7. Yang L, Yang Y, Ma P, et al. Spread through air spaces predicts a worse survival in patients with stage I adenocarcinomas $>2 \mathrm{~cm}$ after radical lobectomy. J Thorac Dis 2019;11:E80-E81.

8. Shiono S, Yanagawa N. Spread through air spaces is a predictive factor of recurrence and a prognostic factor in stage I lung adenocarcinoma. Interact Cardiovasc Thorac Surg 2016;23:567-72.

9. Yi E, Lee JH, Jung Y, et al. Clinical implication of tumour spread through air spaces in pathological stage I lung adenocarcinoma treated with lobectomy. Interact Cardiovasc Thorac Surg 2021;32:64-72.

10. Ren Y, Xie H, Dai C, et al. Prognostic impact of tumor spread through air spaces in sublobar resection for 1A lung adenocarcinoma patients. Ann Surg Oncol 2019;26:1901-8.

11. Uruga H, Fujii T, Fujimori S, et al. Semiquantitative Assessment of Tumor Spread through Air Spaces (STAS) in Early-Stage Lung Adenocarcinomas. J Thorac Oncol 2017;12:1046-51.

12. Dai C, Xie H, Su H, et al. Tumor Spread through Air Spaces Affects the Recurrence and Overall Survival in Patients with Lung Adenocarcinoma $>2$ to $3 \mathrm{~cm}$. J Thorac Oncol 2017;12:1052-60.

13. Suzuki K, Watanabe S, Wakabayashi M, et al. A nonrandomized confirmatory phase III study of sublobar surgical resection for peripheral ground glass opacity dominant lung cancer defined with thoracic thin-section computed tomography (JCOG0804/WJOG4507L). J Clin Oncol 2017;35:Abstract 8561.

14. Aokage K, Yoshida J, Hishida T, et al. Limited resection for early-stage non-small cell lung cancer as functionpreserving radical surgery: A review. Jpn J Clin Oncol 
2017;47:7-11.

15. Hennon M, Landreneau RJ. Role of Segmentectomy in Treatment of Early-Stage Non-Small Cell Lung Cancer. Ann Surg Oncol 2018;25:59-63.

16. Asamura H, Aokage K, Yotsukura M. Wedge Resection Versus Anatomic Resection: Extent of Surgical Resection for Stage I and II Lung Cancer. Am Soc Clin Oncol Educ Book 2017;37:426-33.

17. Kadota K, Nitadori J, Sima CS, et al. Tumor Spread through Air Spaces is an Important Pattern of Invasion and Impacts the Frequency and Location of Recurrences after Limited Resection for Small Stage I Lung Adenocarcinomas. J Thorac Oncol 2015;10:806-14.

18. Ma K, Zhan C, Wang S, et al. Spread Through Air Spaces (STAS):A New Pathologic Morphology in Lung Cancer. Clin Lung Cancer 2019;20:e158-e162.

19. Kadota K, Kushida Y, Kagawa S, et al. Limited Resection Is Associated With a Higher Risk of Locoregional Recurrence than Lobectomy in Stage I Lung Adenocarcinoma With Tumor Spread Through Air Spaces. Am J Surg Pathol 2019;43:1033-41.

Cite this article as: Yang Y, Xie X, Wang Y, Li X, Luo L, Yao Y, Li J. A systematic review and meta-analysis of the influence of STAS on the long-term prognosis of stage I lung adenocarcinoma. Transl Cancer Res 2021;10(5):2428-2436. doi: $10.21037 /$ tcr-21-750
20. Zhong Y, Xu Y, Deng J, et al. Prognostic impact of tumour spread through air space in radiological subsolid and pure solid lung adenocarcinoma. Eur J Cardiothorac Surg 2021;59:624-32.

21. Chen D, She Y, Wang T, et al. Radiomics-based prediction for tumour spread through air spaces in stage I lung adenocarcinoma using machine learning. Eur J Cardiothorac Surg 2020;58:51-8.

22. Warth A, Muley T, Kossakowski CA, et al. Prognostic impact of intra-alveolar tumor spread in pulmonary adenocarcinoma. Am J Surg Pathol 2015;39:793-801.

23. Han YB, Kim H, Mino-Kenudson M, et al. Tumor spread through air spaces (STAS): prognostic significance of grading in non-small cell lung cancer. Mod Pathol 2021;34:549-61. Erratum in: Mod Pathol 2021;34:1038.

24. Toyokawa G, Yamada Y, Tagawa T, et al. Significance of spread through air spaces in resected pathological stage I lung adenocarcinoma. Ann Thorac Surg 2018;105:1655-63.

(English Language Editor: B. Draper) 